Cahiers $d u$ MONDE RUSSE

\section{Cahiers du monde russe}

Russie - Empire russe - Union soviétique et États indépendants

$47 / 4 \mid 2006$

Varia

\title{
Christopher Andrew, Vasili Mitrokhin, The World Was Going Our Way
}

Andreï Kozovoï

\section{OpenEdition}

\section{Journals}

Édition électronique

URL : https://journals.openedition.org/monderusse/6776

DOI : 10.4000/monderusse. 6776

ISSN : $1777-5388$

Éditeur

Éditions de l'EHESS

\section{Édition imprimée}

Date de publication : 30 décembre 2006

Pagination : $903-906$

ISBN : 978-2-7132-2098-2

ISSN : $1252-6576$

Référence électronique

Andreï Kozovoï, « Christopher Andrew, Vasili Mitrokhin, The World Was Going Our Way », Cahiers du monde russe [En ligne], 47/4 | 2006, mis en ligne le 03 juillet 2009, consulté le 03 septembre 2022. URL : http://journals.openedition.org/monderusse/6776; DOI : https://doi.org/10.4000/monderusse. 6776

Ce document a été généré automatiquement le 3 septembre 2022.

Tous droits réservés 


\title{
Christopher Andrew, Vasili Mitrokhin, The World Was Going Our Way
}

\author{
Andreï Kozovoï
}

\section{RÉFÉRENCE}

Christopher ANDREW, Vasili MITROKHIN, The World Was Going Our Way. The KGB

and the Battle for the Third World. New York : Basic Books ; Londres : Allen Lane, 2005, $676 \mathrm{p}$.

1 Après deux volumes consacrés à la politique secrète du KGB dans le monde, Christopher Andrew, professeur à Cambridge et spécialiste reconnu de la question, publie avec l'aide du transfuge Vasilij Mitrohin (1922-2004) un ouvrage centré sur le Tiers-Monde. Rappelons qu'Andrew s'est rendu célèbre en publiant en 1990 KGB : The Inside Story of Its Foreign Operations from Lenin to Gorbachev, à partir des souvenirs de l'ancien colonel du KGB Oleg Gordievskij, exfiltré en 1985 après onze ans de bons et loyaux services pour le MI-6 ${ }^{1}$. Le témoignage de Mitrohin s'avère bien plus important, aussi bien quantitativement que qualitativement. Exfiltré en 1992 par les Britanniques² ${ }^{2}$, sa présence à l'Ouest est gardée secrète jusqu'à la publication en 1999 du best-seller international The Sword and the Shield. The Mitrokhin Archive and the Secret History of the KGB'. Responsable des archives du KGB depuis 1972, Mitrohin prend des notes sur des bouts de papier jusqu'en 1984, date de son départ à la retraite. Les informations qu'il transmet aux Britanniques permettent, entre autres, de démasquer la doyenne des espionnes, Melita Norwood (1912-2005), qui travaille pour Moscou depuis 1937 (!).

2 The Sword and the Shield révélait les opérations secrètes du KGB en Europe et aux ÉtatsUnis. Le présent volume se concentre sur l'Amérique latine, le Moyen-Orient, l'Asie et l'Afrique pour mettre au jour la stratégie globale du Kremlin consistant à entretenir et à développer l'antiaméricanisme « naturel » dans ces pays. Pour Andrew et Mitrohin, l'idée 
que la guerre froide pouvait être gagnée dans le Tiers-Monde était bien plus ancrée chez les chefs du KGB que chez les autres responsables du Kremlin.

Le plan adopté par l'auteur est géographique. En Amérique latine, entre autres exemples, les notes de Mitrohin confirment le soutien à Castro des Américains de la « Venceremos Brigade ", utilisés dans un but de propagande à partir de 1969, mais qui ont aussi fourni des papiers d'identité américains pour des opérations secrètes (p. 55-56). Ailleurs, Andropov, chef du KGB à partir de 1967, préfère recruter des " contacts confidentiels ", dont la mission est d'influencer la politique de leurs gouvernements à l'égard des ÉtatsUnis dans un sens favorable à Moscou. La stratégie appliquée au Pérou est particulièrement révélatrice (p. 62-65). Pour ce qui est des financements, les dirigeants latino-américains acceptent assez souvent l'argent de Moscou et jurent fidélité aux idées du marxisme-léninisme, pour n'agir ensuite qu'à leur guise. L'exemple de José Figueras Ferrer, un social-démocrate du Costa Rica, est éloquent : financé par le KGB, il n'en demeure pas moins un anticommuniste convaincu (p. 67-69). La question du financement d'Allende reçoit une ultime confirmation: l'argent de Moscou fut envoyé de manière quasi continue, agrémenté de cadeaux, dont notamment des icônes (p.71-80). Après la mort du Chilien, c'est le KGB qui se charge d'en forger une image de martyr, tant dans les médias soviétiques qu'à l'étranger; ce qu'il tentera de faire également, avec beaucoup moins de succès, dans le cas de Luis Corvalan (p. 85-86).

Le coup d'État de Pinochet sert de prétexte aux Soviétiques pour réactualiser leur stratégie générale vis-à-vis de l'Amérique latine. À cet égard, la réunion de février 1974 du Politbjuro apparaît essentielle et vient combler une lacune importante dans notre connaissance du processus décisionnel soviétique, tout en rappelant opportunément que la politique étrangère de l'URSS n'était pas que simple réaction aux mouvements des Américains, sans plan d'ensemble coordonné ${ }^{4}$. Enfin, les documents de Mitrohin montrent bien le soutien du KGB aux sandinistes du Nicaragua (p.116-117) et aux révolutionnaires du Salvador (p. 123-125), tout en laissant à Castro le rôle le plus visible : il s'agit de pas aggraver les relations avec l'Occident après les retombées de l'invasion de l'Afghanistan.

5 Si l'Amérique latine est « l'arrière-cour » des États-Unis, le Moyen-Orient est celle de l'URSS - c'est du moins ce que pensent les responsables soviétiques. Ceci les incite à plus d'optimisme que dans le cas précédent. Concernant l'Égypte, ce bel optimisme finit cependant par s'effondrer lorsque Sadate se rapproche des États-Unis, dès 1972. Les tentatives du KGB pour lui faire croire que Kissinger lui tend un piège ayant échoué, Andropov monte une affaire qui tente de discréditer le secrétaire égyptien aux Affaires étrangères, Ashraf Marwan. Le remplacement de celui-ci en 1976 fait penser au KGB que l'opération a réussi (p. 160-161). Autre exemple: pour contrer l'influence américaine en Iran, l'URSS se concentre sur l'Irak en exploitant les dissensions entre le régime baasiste et l'opposition, entre autres kurde. De fait, Moscou pratique une politique cynique d'alliance avec Saddam Hussein et de soutien à l'opposition communiste kurde à partir de 1979 (p. 188). Finalement, le seul véritable allié des Soviétiques dans la région, si l'on excepte la République populaire du Yémen, semble avoir été la Syrie d'Hafez el-Assad: malgré l'interdiction du parti communiste, Moscou lui livre armes et conseillers militaires à foison (p. 195). En ce qui concerne Israël, on découvre le détail de plusieurs opérations destinées à discréditer les sionistes. Ainsi, lors de l'opération « SIMON » en février 1973, une liste d'un millier de noms de sympathisants français est volée à Paris 
(p. 238). Enfin, les liens entre le KGB et les terroristes palestiniens sont mis en évidence (p. 246 sqq.).

6 En Asie, les méthodes sont les mêmes qu'ailleurs, même si le KGB rencontre bien des difficultés à infiltrer les milieux politiques chinois et japonais. Le pays sur lequel il concentre l'essentiel de ses efforts est l'Inde. Mais, malgré les très nombreuses « opérations actives » destinées à influencer les élections, Indira Gandhi subit une défaite en 1977. Les opérations continuent cependant au cours des années 1980, notamment dans le but de rendre responsables la CIA et les services de renseignement pakistanais des mouvements séparatistes sikhs du Penjab (p. 336 sqq.). En 1980, Andropov approuve une série de mesures destinées à empêcher le général Zia du Pakistan de favoriser l'aide des Américains (et des Chinois) aux résistants afghans (p. 355-358). Enfin, la plupart des notes de Mitrohin, prises entre 1978 et 1983, concernant l'Afghanistan, et publiées par le Cold War International History Project, sont reprises dans les deux derniers chapitres portant sur les opérations dans ce pays, ce qui relativise l'apport de l'ouvrage pour les lecteurs les plus avertis 5 .

7 Le dernier continent à être traité, l'Afrique, est un vaste champ de bataille qui a connu plusieurs victoires soviétiques. Entre autres exemples, Andrew mentionne le stratagème du KGB dans les années 1960, consistant à envoyer aux délégués africains de l'ONU des lettres provenant de prétendus racistes américains (p.436). En Guinée, de faux documents dévoilant un « complot de la CIA » produisent leur effet sur Sékou Touré, qui expulse les officiers américains. Même scénario au Nigeria, pourtant bien moins «progressiste» (p. 437-439). Enfin, dans le cas de l'Angola et du Mozambique, pays qui suscitent les plus grands espoirs du Kremlin au milieu des années 1970, les notes de Mitrohin se contentent simplement d'identifier les agents soviétiques ayant infiltré les partis en place.

8 La question centrale qu'un historien de l'URSS est amené à se poser en présence de ce livre est naturellement celle de la validité, puis du choix des sources, puis globalement la question de l'apport d'un ouvrage destiné d'abord à un public de non-spécialistes. Le lecteur circonspect doit se référer continuellement aux notes en fin de volume, afin de distinguer l'apport de Mitrohin de ce qui est déjà publié. Globalement, à l'exception de plusieurs notes essentielles comme celles qui portent sur l'invasion de l'Afghanistan, l'ensemble ne fait que corroborer ce que l'on savait déjà et, le plus souvent, n'ajoute que le détail des financements et le nom des agents. À la différence du volume précédent, il n'y a pas ici de révélations « fracassantes ». Ceci explique sans doute que la commission chargée de vérifier le contenu avant publication n'ait censuré que deux pages du manuscrit (les notes de Mitrohin ne sont pas accessibles au public) (p. XXXII-XXXIII).

9 Mais surtout, en accord avec la position de Mitrohin lui-même, que l'auteur s'efforce pourtant de nuancer (ibid.), le livre adopte un point de vue trop souvent centré sur le KGB. Les réunions et/ou les autorisations du sommet (du Politbjuro en particulier) sont rarement mentionnées, et le KGB semble le plus souvent agir de manière autonome. L'historien de l'URSS doit donc rester prudent devant ce qui pourrait au fond ne constituer qu'un tableau assez biaisé du fonctionnement décisionnel soviétique. En outre, comme l'indique le titre de l'ouvrage (qu'on pourrait traduire par « le monde était à nos pieds »), le KGB apparaît triomphant. Or, le contexte économique général n'incitait guère au triomphalisme dans les années brejnéviennes. Le seul moment où l'optimisme des employés du KGB connut son apogée fut la période 1975-1976, lors du « repli » de l'Amérique. En l'état actuel de nos connaissances et des archives disponibles - les fonds 
du KGB pour les années les plus récentes sont inaccessibles au commun des mortels -, l'historien ne peut juger de la représentativité des notes. Quoi qu'il en soit, et en dépit de ces réserves, dans le contexte d'une hypermémoire des « activités néfastes » de la CIA, la publication du deuxième volume des « archives Mitrohin " permet de rappeler dans le détail le déroulement des pratiques secrètes du régime totalitaire soviétique et de rééquilibrer la donne.

\section{NOTES}

1. Christopher Andrew, Oleg Gordievsky, Le KGB dans le monde, 1917-1990, P. : Fayard, 1990, 750 p.

2. Il s'était auparavant adressé à l'ambassade américaine, mais le résident de la CIA n'avait pas prêté attention à sa demande, pensant qu'il s'agissait d'un bibliothécaire, et non d'un espion.

3. Christopher Andrew, Vassili Mitrokhine, Le KGB contre l'Ouest, 1917-1991, P. : Fayard, 2001, $982 \mathrm{p}$.

4. Voir p. 89. Un précédent existe: en juillet 1961, un "plan global» de lutte contre « l'adversaire principal» (les États-Unis) et ses alliés, dans le but de faciliter le règlement de la question allemande en faveur des Soviétiques, fut élaboré par Šelepin et envoyé au Comité central, qui le valida (voir p. 40).

5. Voir le Working paper $n^{\circ} 40$ (février 2002) du CWIHP, "The KGB in Afghanistan ", à l'adresse suivante :

http://www.wilsoncenter.org/index.cfm?

topic_id=1409\&fuseaction=topics.publications\&group_id=11901. 